\title{
Azathioprine with Allopurinol Is a Promising First-Line Therapy for Inflammatory Bowel Diseases
}

\author{
Elsa L. S. A. van Liere ${ }^{1,2}$ (1) $\cdot$ Ahmed B. Bayoumy ${ }^{1} \cdot$ Chris J. J. Mulder ${ }^{3} \cdot$ Ben Warner $^{4} \cdot$ Bu Hayee $^{5} \cdot$ Bilal A. Mateen ${ }^{5} \cdot$ \\ Jonathan D. Nolan ${ }^{2}$. Nanne K. H. de Boer ${ }^{3}$ Simon H. C. Anderson ${ }^{4} \cdot$ Azhar R. Ansari $^{2}$
}

Received: 29 January 2021 / Accepted: 1 October 2021 / Published online: 2 November 2021

(c) The Author(s) 2021

\begin{abstract}
Background Beneficial response to first-line immunosuppressive azathioprine in patients with inflammatory bowel disease (IBD) is low due to high rates of adverse events. Co-administrating allopurinol has been shown to improve tolerability. However, data on this co-therapy as first-line treatment are scarce.

Aim Retrospective comparison of long-term effectiveness and safety of first-line low-dose azathioprine-allopurinol cotherapy (LDAA) with first-line azathioprine monotherapy (AZAm) in patients with IBD without metabolite monitoring.

Methods Clinical benefit was defined as ongoing therapy without initiation of steroids, biologics or surgery. Secondary outcomes included CRP, HBI/SCCAI, steroid withdrawal and adverse events.

Results In total, 166 LDAA and 118 AZAm patients (median follow-up 25 and 27 months) were evaluated. Clinical benefit was more frequently observed in LDAA patients at 6 months ( $74 \%$ vs. $53 \%, p=0.0003), 12$ months $(54 \%$ vs. $37 \%, p=0.01)$ and in the long-term (median 36 months; $37 \%$ vs. $24 \%, p=0.04$ ). Throughout follow-up, AZAm patients were $60 \%$ more likely to fail therapy, due to a higher intolerance rate $(45 \%$ vs. $26 \%, p=0.001)$. Only $73 \%$ of the effective AZA dose was tolerated in AZAm patients, while LDAA could be initiated and maintained at its target dose. Incidence of myelotoxicity and elevated liver enzymes was similar in both cohorts, and both conditions led to LDAA withdrawal in only $2 \%$. Increasing allopurinol from 100 to 200-300 mg/day significantly lowered liver enzymes in 5/6 LDAA patients with hepatotoxicity. Conclusions Our poor AZAm outcomes emphasize that optimization of azathioprine is needed. We demonstrated a longterm safe and more effective profile of first-line LDAA. This co-therapy may therefore be considered standard first-line immunosuppressive.
\end{abstract}

Keywords Azathioprine · Allopurinol · Thiopurines · Inflammatory bowel disease · Drug repositioning

\section{Introduction}

Azathioprine (AZA) is a cornerstone immunosuppressive therapy for patients with inflammatory bowel disease (IBD) $[1,2]$. It improves important outcomes such as hospitalization, surgery, steroid use and colorectal cancer risk [3-7]. Moreover, AZA has been used for over 50 years in millions of patients worldwide and hence common as well as rare adverse events are well documented. As IBD most commonly affects patients during their reproductive years, the well-established safety of AZA during pregnancy and

Elsa L. S. A. van Liere

elsa.vanliere@amsterdamumc.nl

Extended author information available on the last page of the article breastfeeding is an important advantage, as compared to newer biologics and small-molecule drugs [8-10].

Unfortunately, AZA is limited by adverse events with the tolerated dose often being well below the effective dose [11, 12], resulting in 55\% of patients discontinuing therapy within 5 years due to intolerance or ineffectiveness [13]. Consequently, a shift towards early therapy with biologics has occurred in those in more affluent countries. Whilst biologics can be transformative, they do have limitations: poor long-term effectiveness (related to antibody formation) [14]; parenteral administration, which affects quality of life [15]; infusion reactions (occasionally life-threatening) [16]; and high costs [17]. To address some of these concerns, new small-molecule drugs are being developed. However, the potential to optimize established small-molecule drugs like the thiopurines, and subsequently reduce the number 
of patients escalated to treatments like biologics, is often overlooked. Since thiopurines are affordable this strategy also reduces healthcare costs, which is particularly pertinent for developing countries and uninsured patients in higherincome countries [18].

One such optimization strategy for patients with IBD is to combine low-dose thiopurine with allopurinol, which was first described in 2005 [19]. Despite the fact that switching AZA monotherapy (AZAm) to low-dose AZA with allopurinol co-therapy (LDAA) improves tolerability and efficacy [19-26], LDAA remains underreported in current literature [27], not mentioned in most IBD guidelines [1,2] and hence underutilized. Moreover, most studies restricted LDAA to patients both failing AZA (due to intolerance or inefficacy) and showing aberrant metabolism i.e. hypermethylation (production of toxic 6-MMPs at the expense of therapeutic 6-TGNs). Conversely, several studies have demonstrated a poor predictive value of metabolites with regard to clinical outcomes. For instance, 52\% of the patients resistant [28] and up to $50 \%$ of the patients intolerant to AZAm [26, 29] were non-hypermethylators. Of those with high 6-MMP levels, nearly $90 \%$ did not show hepatotoxicity $[29,30]$. Only using LDAA in patients showing hypermethylation thus excludes a large number of patients who could benefit from LDAA. Furthermore, this approach hinders LDAA use in less affluent institutions/countries without access to metabolite measurements.

Apart from being a valuable option for all patients with IBD who have failed AZAm, LDAA might be beneficial as first-line immunosuppressive therapy [24]. Using LDAA as first-line therapy in daily practice (rather than AZAm) could reduce delay in reaching an effective treatment, and could eliminate complex drug dosing and extra monitoring typically needed with AZAm (LDAA can be initiated and maintained at the target/effective dose in most patients). This would improve both patient and clinician experience.

Hence, we sought to evaluate the long-term effectiveness and safety of first-line LDAA therapy in a large IBD cohort undirected by 6 -TGNs/6-MMPs, and to compare outcomes with a similar AZAm cohort.

\section{Methods}

\section{Study Design}

Medical records of a single centre (East Surrey Hospital, UK) were retrospectively reviewed. Subjects were identified by cross-referencing hospital pharmacy dispensing records from $01 / 2014$ to $05 / 2019$ with a prospective patient registry. Patients with Crohn's disease, ulcerative colitis or IBDunclassified and treated with either LDAA or AZAm as first thiopurine therapy, were included. Patients below 18 years, without complete follow-up or with commencement of therapy before 2014 (to avoid overlap with previously published data [24]) were excluded. Patients were followed until thiopurine discontinuation or therapy escalation, or May 2019.

Throughout the study period, patients were allocated to either LDAA or AZAm based on patient's preference and/or clinician's experience/preference with regard to these treatments. AZAm patients were initiated on $50 \mathrm{mg} /$ day, slowly increased to a target dose of $2.5 \mathrm{mg} / \mathrm{kg} /$ day or $1.25 \mathrm{mg} / \mathrm{kg} /$ day in case of wild-type or heterozygous TPMT activity, respectively. LDAA patients received a target dose from the beginning, i.e. $25 \%$ of the equivalent AZAm dose. Allopurinol was commenced at $100 \mathrm{mg} /$ day.

Hematological, biochemical and inflammatory parameters were monitored every 2 weeks for the first 12 weeks and then every 3 months. 6-TGNs and 6-MMPs were not routinely measured.

This study was considered an audit of routine clinical practice, hence was qualified as being exempt from Research Ethical Committee review. The study was conducted in accordance with the Declaration of Helsinki.

\section{Effectiveness}

Primary outcome was clinical benefit, which was defined as ongoing therapy without initiation of corticosteroids, biologics or bowel resection, and thus incorporated both efficacy and tolerability. Clinical benefit was assessed at 6 and 12 months. Moreover, sustained clinical benefit was assigned when the criterion was met during at least 12 months and maintained until final follow-up. Secondary outcomes included IBD activity scores, CRP and withdrawal of concomitant IBD medication.

\section{Safety}

Adverse events and reasons for withdrawal were recorded. Liver abnormalities were categorized into hepatotoxicity (ALT $>$ twofold the upper limit of normal; $>82 \mathrm{U} / \mathrm{L}$ ) and elevated liver enzymes without hepatotoxicity (ALT 41-82 $\mathrm{U} / \mathrm{L})$. Leukopenia was defined as leukocytes $<3.5 \times 10^{9} / \mathrm{L}$ and thrombocytopenia as platelets $<150 \times 10^{9} / \mathrm{L}$.

\section{Statistics}

Effectiveness analyses were performed according to the perprotocol principle. Patients requiring steroids/biologics during the first three months were excluded from the effectiveness analysis, considering that AZA requires three months to become fully effective [31].

Chi-square and Mann-Whitney U tests were used to compare outcomes between groups. A Kaplan-Meier plot visualized survival characteristics (event: non-clinical 
benefit); the curves were compared using Mantel-Cox test and a Cox-regression yielded the hazard ratio.

Patient characteristics associated with clinical benefit in the LDAA cohort based on a univariable Coxregression $(p<0.20)$ were included in a multivariable model to identify independent associated factors. A univariate logistic regression examined possible correlations between intolerance to LDAA and gender, age, IBD-type, TPMT activity.

Statistical analyses were performed using IBM SPSS Statistics version 25. A $p$-value $<0.05$ was considered statistically significant.

\section{Results}

\section{Patient and Therapy Characteristics}

In total, 166 LDAA and 118 AZAm patients were included (Fig. 1), with a median follow-up of 25 and 27 months, respectively. Collected baseline characteristics were very similar in both cohorts (Table 1). Primary indication for thiopurines was 'active disease' (LDAA $n=149$ [90\%]; AZAm $n=109$ [92\%]). All patients were thiopurine-naïve and most patients were immunosuppressant-naïve (150 LDAA [90\%] and 113 AZAm [97\%]).

Only 14 (8\%) LDAA and 3 (3\%) AZAm patients used concurrent biologic. In most cases (11/14 LDAA and 2/3 AZAm patients) thiopurine and biologic therapy were commenced simultaneously because of a top-down therapeutic approach. In those with normal TPMT activity ( 90\%), median AZA dose was $0.54 \mathrm{mg} / \mathrm{kg} /$ day (LDAA) and $1.83 \mathrm{mg} / \mathrm{kg} /$ day (AZAm).

\section{Effectiveness}

Clinical benefit rates are summarized in Figs. 2 and 3, and Table 2. Clinical benefit was more frequently observed in LDAA than AZAm patients at 6 months (116/156 [74\%] vs. 59/111 [53\%], $p=0.0003)$ and 12 months (74/138 [54\%] vs. $38 / 103$ [37\%], $p=0.01$ ). Furthermore, 51/138 (37\%) LDAA compared to 25/103 (24\%) AZAm subjects demonstrated sustained clinical benefit during a median of 36 (IQR 20-44) and 33 (IQR 22-44) months, respectively ( $p=0.04$ ).

A Kaplan-Meier curve (Fig. 4) demonstrates that more LDAA than AZAm patients were having clinical benefit during the entire study period $(p=0.003)$. Throughout follow-up, AZAm individuals were $60 \%$ more likely to fail therapy (95\%-CI 1.16-2.14). Moreover, median duration of beneficial response from commencement of therapy was 17 months in the LDAA cohort (95\%-CI 9-25) compared to 6 months in the AZAm cohort (95\%-CI 1-11).

A multivariable analysis on the association between patient characteristics and clinical benefit in LDAA-treated patients (Table S1, Supplementary Material) demonstrated that stricturing Crohn's disease was inversely correlated with clinical benefit (hazard ratio 2.09, 95\%-CI 1.02-4.28). No difference in response to LDAA was found between Crohn's disease and ulcerative colitis patients, nor between patients with and without concurrent biologic therapy.

\section{Secondary Outcomes}

Secondary outcomes are summarized in Table 3. Steroid withdrawal within 6 months occurred in approximately $90 \%$ of both cohorts. In the LDAA cohort, 65\% (69/106) demonstrated clinical remission ( $\mathrm{SCCAI} \leq 2$ or $\mathrm{HBI} \leq 4)$ at last review, compared to 58\% (45/78) in the AZAm $(p=0.31)$. Biochemical remission (CRP $\leq 10 \mathrm{mg} / \mathrm{L}$ ) was observed
Excluded $(n=232)$

No patient file available, $n=4$

Absence of IBD, $n=3$

Incomplete follow-up, $\mathrm{n}=18$

No commencement of therapy, $\mathrm{n}=14$

Commencement of therapy before 2014, $n=98$

History of thiopurine use, $\mathrm{n}=95$

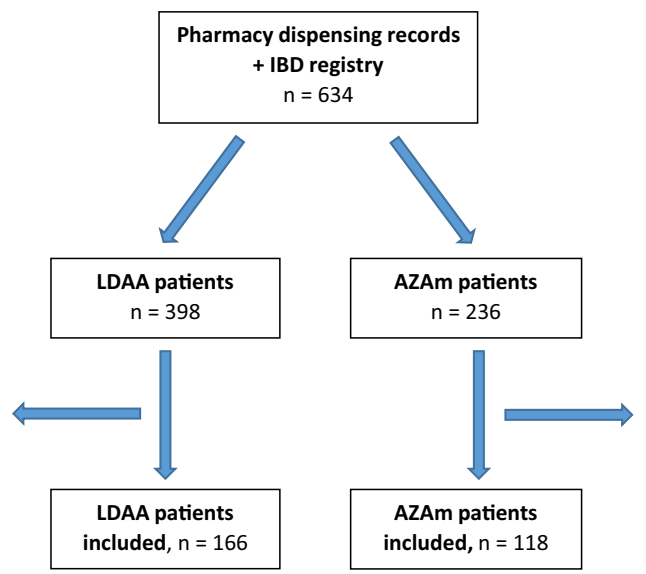

Fig. 1 Flowchart showing the selection of the study population 
Table 1 Patient and disease characteristics, $n(\%)$ or median (IQR)

\begin{tabular}{|c|c|c|}
\hline & LDAA patients $(n=166)$ & AZAm patients $(n=118)$ \\
\hline Female & $95(57 \%)$ & $65(55 \%)$ \\
\hline \multicolumn{3}{|l|}{ Smoking } \\
\hline Yes & $20(12 \%)$ & $17(14 \%)$ \\
\hline No & $65(39 \%)$ & $50(42 \%)$ \\
\hline Unknown & $81(49 \%)$ & $51(43 \%)$ \\
\hline Age at initiation, $y$ & $41(29-55)$ & $35(27-51)$ \\
\hline IBD duration at initiation, $y$ & $1(1-6)$ & $1(0-3)$ \\
\hline Crohn's disease & $93(56 \%)$ & $63(53 \%)$ \\
\hline \multicolumn{3}{|l|}{ Age at diagnosis, $y$} \\
\hline$<17$ & $3(3 \%)$ & $2(3 \%)$ \\
\hline $17-40$ & $53(57 \%)$ & $42(67 \%)$ \\
\hline$>40$ & $34(37 \%)$ & $15(23 \%)$ \\
\hline Unknown & $3(3 \%)$ & $4(6 \%)$ \\
\hline \multicolumn{3}{|l|}{ Behaviour } \\
\hline Inflammatory & $47(50 \%)$ & $39(62 \%)$ \\
\hline Stricturing & $26(28 \%)$ & $20(32 \%)$ \\
\hline Penetrating & $10(11 \%)$ & $2(3 \%)$ \\
\hline Unknown & $10(11 \%)$ & $2(3 \%)$ \\
\hline Perianal disease & $12(13 \%)$ & $8(13 \%)$ \\
\hline \multicolumn{3}{|l|}{ Location } \\
\hline Ileal & $33(35 \%)$ & $30(48 \%)$ \\
\hline Colonic & $26(28 \%)$ & $18(29 \%)$ \\
\hline Ileocolonic & $29(31 \%)$ & $14(22 \%)$ \\
\hline Unknown & $5(5 \%)$ & $1(2 \%)$ \\
\hline Ulcerative colitis & $72(43 \%)$ & $52(44 \%)$ \\
\hline \multicolumn{3}{|l|}{ Extent } \\
\hline Proctitis & $9(13 \%)$ & $4(8 \%)$ \\
\hline Left-sided & $30(42 \%)$ & $25(48 \%)$ \\
\hline Pancolitis & $27(38 \%)$ & $19(37 \%)$ \\
\hline Unknown & $6(8 \%)$ & $4(8 \%)$ \\
\hline IBD unclassified & $1(1 \%)$ & $3(3 \%)$ \\
\hline Bowel resection history & $15(9 \%)$ & $10(9 \%)$ \\
\hline \multicolumn{3}{|l|}{ Prior drug failure } \\
\hline None & $40(24 \%)$ & $24(21 \%)$ \\
\hline Aminosalicylates & $119(72 \%)$ & $93(79 \%)$ \\
\hline Methotrexate & $2(1 \%)$ & 0 \\
\hline Calcineurin inhibitor & $9(5 \%)$ & $4(3 \%)$ \\
\hline Anti-tumour necrosis factor & $5(3 \%)$ & $1(1 \%)$ \\
\hline \multicolumn{3}{|l|}{ TPMT activity } \\
\hline Wild type range $^{\mathrm{a}}$ & $137(87 \%)$ & $100(90 \%)$ \\
\hline Heterozygous range ${ }^{\mathrm{b}}$ & $21(13 \%)$ & $11(9 \%)$ \\
\hline Unknown & $8(5 \%)$ & $7(6 \%)$ \\
\hline \multicolumn{3}{|c|}{ Indication for thiopurine therapy } \\
\hline Active disease $^{c}$ & $149(90 \%)$ & $109(92 \%)$ \\
\hline Intolerance to prior therapy & $3(2 \%)$ & $3(3 \%)$ \\
\hline Optimisation of biologics & $2(1 \%)$ & $1(1 \%)$ \\
\hline High risk profile ${ }^{c}$ & $4(2 \%)$ & $1(1 \%)$ \\
\hline Unknown & $8(5 \%)$ & $4(3 \%)$ \\
\hline \multicolumn{3}{|c|}{ IBD $^{\text {activity scores at initiation }}{ }^{\mathrm{d}}$} \\
\hline SCCAI & $6(3-7)$ & $5(3-6)$ \\
\hline HBI & $5(3-8)$ & $7(5-10)$ \\
\hline
\end{tabular}


Table 1 (continued)

\begin{tabular}{lll}
\hline & LDAA patients $(n=166)$ & AZAm patients $(n=118)$ \\
\hline Azathioprine dose at final follow- up in $m g / k g /$ day & \\
Entire cohort & $0.52(0.43-0.58)$ & $1.75(1.17-2.11)$ \\
TPMT wild type ${ }^{\mathrm{a}}$ patients & $0.54(0.47-0.59)$ & $1.83(1.54-2.18)$ \\
TPMT heterozygous ${ }^{\mathrm{b}}$ patients & $0.35(0.30-0.43)$ & $0.93(0.80-1.14)$ \\
Concomitant IBD medication & & \\
None & $41(25 \%)$ & $34(29 \%)$ \\
Aminosalicylates & $83(50 \%)$ & $61(52 \%)$ \\
Calcineurin inhibitor & $7(4 \%)$ & $2(2 \%)$ \\
Anti-tumour necrosis factor & $14(8 \%)$ & $3(3 \%)$ \\
Steroids $\geq 10$ mg/day & $53(32 \%)$ & $44(37 \%)$ \\
Follow-up time, $m$ & $25(13-43)$ & $27(14-42)$ \\
\hline
\end{tabular}

$L D A A$ low-dose azathioprine with allopurinol, $A Z A m$ azathioprine monotherapy, $I B D$ inflammatory bowel disease, SCCAI Simple Clinical Colitis Activity Index, HBI Harvey-Bradshaw Index, TPMT thiopurine s-methyltransferase

${ }^{\mathrm{a}} 68-150 \mathrm{mU} / \mathrm{L}$

b $20-67 \mathrm{mU} / \mathrm{L}$

${ }^{\mathrm{c}}$ In accordance with international IBD guidelines[10]

${ }^{\mathrm{d}}$ Disease activity scores (SCCAI for ulcerative colitis or HBI for Crohn's disease) at initiation were documented in the patients' medical records in 85/166 (51\%) LDAA and 63/118 (53\%) AZAm patients

in $93 / 158(59 \%)$ and $59 / 94(63 \%)$ of LDAA and AZAm patients, respectively $(p=0.54)$.

\section{Adverse Events}

Sixty-eight of the 116 AZAm (58\%) and 80/166 (48\%) LDAA patients experienced adverse events during a median follow-up of 27 and 25 months, respectively ( $p=0.12$, Table 4). Adverse events were the main reason for discontinuing either LDAA or AZAm. However, a greater proportion of AZAm patients discontinued due to adverse events (AZAm: 53/118 [45\%]; LDAA: 43/166 [26\%]; $p=0.001$ ). LDAA withdrawal due to intolerance did not depend on gender, age, IBD-type or TPMT level.

No malignancies were reported in either cohort and the incidence of pancreatitis was low.

\section{Liver Enzyme Abnormalities}

Liver enzyme abnormalities occurred equally in both cohorts: $11(7 \%)$ patients reported hepatotoxicity and 13 (8\%) elevated liver function tests (LFTs) without hepatotoxicity in the LDAA cohort, compared to 8 (7\%) and $10(8 \%)$ AZAm patients, respectively. Upon more detailed review of LDAA patients, 4/11 reporting hepatotoxicity and 5/13 reporting elevated LFTs without hepatotoxicity had liver disease prior to LDAA initiation (fatty liver $n=7$; focal nodular hyperplasia $n=1$; primary sclerosing cholangitis $n=1$ ). In contrast, 1/18 AZAm patients with liver enzyme abnormalities had prior liver disease (fatty liver).
Hepatotoxicity led to LDAA discontinuation in two (1\%) patients as did elevated LFTs without hepatotoxicity in one patient $(0.6 \%)$. In six other patients with hepatotoxicity, allopurinol dose was increased to $200-300 \mathrm{mg}$ /day. Consequently, the abnormal LFTs resolved in $4 / 6$, improved in $1 / 6$ and persisted in the remaining patient (who had primary sclerosing cholangitis). All six patients continued LDAA until final follow-up and did not experience further side effects on the escalated allopurinol dose.

AZAm was discontinued due to hepatotoxicity and elevated LFTs without hepatotoxicity in three (3\%) and two (2\%) patients, respectively.

\section{Myelotoxicity}

Incidence of myelotoxicity was similar in both cohorts (LDAA: 18/166 [11\%]; AZAm: 11/118 [9\%], $p=0.17$ ). One patient in each cohort required hospital admission related to myelotoxicity (LDAA: respiratory tract infection, AZAm: CMV reactivation).

Regarding the LDAA cohort, 2/21 (10\%) with heterozygous TPMT activity developed myelotoxicity, whereas 16/137 (12\%) patients with wild-type TPMT $(p=0.77)$. Additionally, myelotoxicity was not related to gender, age or IBD-type.

Upon minor AZA dose reduction in 5/12 LDAA patients with leukopenia, leukocyte count normalized in three patients. All three were able to continue therapy until final follow-up, without loss of response. Myelotoxicity resulted in permanent LDAA withdrawal in 3/166 (2\%) patients. 


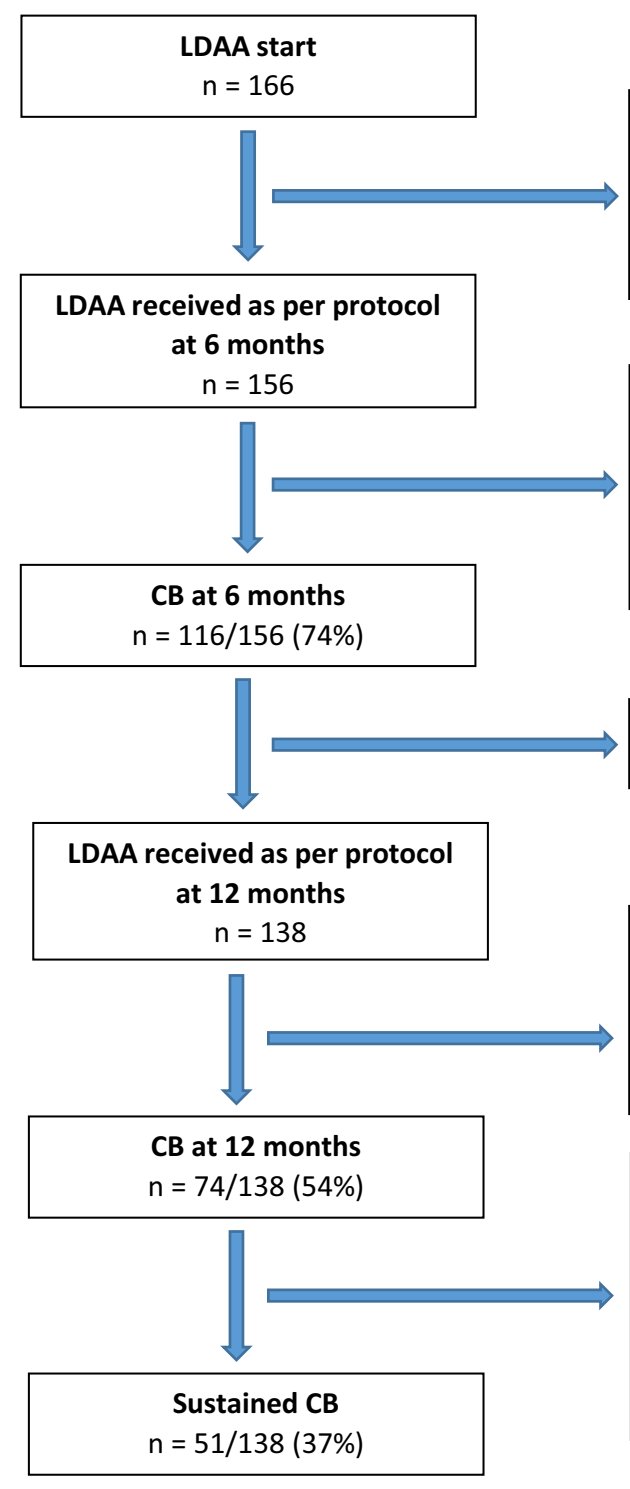

\begin{tabular}{|ll|}
\hline Protocol breaks, $\mathrm{n}=\mathbf{1 0}$ & \\
Steroid course within 3 months & $\mathrm{n}=5$ \\
Biological initiation within 3 months & $\mathrm{n}=3$ \\
Planned resection & $\mathrm{n}=1$ \\
Not yet taking treatment for 6 months & $\mathrm{n}=1$ \\
\hline
\end{tabular}

\begin{tabular}{|ll|}
\hline Non CB, $\mathrm{n}=\mathbf{4 0}$ & \\
Steroid course & $\mathrm{n}=1$ \\
Biological initiation & $\mathrm{n}=3$ \\
IBD-surgery & $\mathrm{n}=1$ \\
Discontinuation due to intolerance $^{\text {Discontinuation due to other reason }}{ }^{1}$ & $\mathrm{n}=32$ \\
\hline
\end{tabular}

Protocol breaks, $\mathrm{n}=18$

Not yet taking treatment for 12 months $\quad n=18$

$\begin{array}{ll}\text { Non CB, } \mathrm{n}=\mathbf{2 4} & \\ \text { Steroid course } & \mathrm{n}=8 \\ \text { Biological initiation } & \mathrm{n}=5 \\ \text { IBD-surgery } & \mathrm{n}=3 \\ \text { Discontinuation due to intolerance } & \mathrm{n}=8\end{array}$

$\begin{array}{ll}\text { Non CB, } \mathbf{n = 2 3} & \\ \text { Steroid course } & n=10 \\ \text { Biological initiation } & n=3 \\ \text { IBD-surgery } & n=2 \\ \text { Discontinuation due to intolerance } & n=3 \\ \text { Discontinuation due to other reason }{ }^{2} & n=2 \\ \text { Discontinuation due to sustained remission } & n=3\end{array}$

1: insufficient response $n=1$, patients' fear $n=2 ; 2$ : insufficient response $n=1$, patients' fear $n=1$

Fig. 2 Clinical benefit (CB) in patients receiving low-dose azathioprine with allopurinol (LDAA)

None of the AZAm patients had a dose reduction after myelotoxicity was detected and 5/118 (4\%) permanently discontinued AZAm due to myelotoxicity.

\section{Discussion}

This is the largest study with the longest follow-up evaluating first-line LDAA for IBD to date, and the first study comparing safety and effectiveness of LDAA $(n=166)$ with AZAm $(n=118)$ in two very similar cohorts. Ongoing therapy without need for steroids, biologics or surgery was more frequently observed in LDAA compared to AZAm patients at 6 months (74\% vs. 53\%), 12 months (54\% vs. $37 \%$ ) and in the long-term (median 36 months, 37\% vs.
24\%). Throughout follow-up, AZAm patients were $60 \%$ more likely to fail therapy, due to a higher intolerance rate (45\% vs. $26 \%$ ).

Direct comparison of our results with others is challenging since most studies have evaluated either effectiveness or tolerability of thiopurines, whereas our primary outcome assessed both elements. Nevertheless, high success rates have been reported for low-dose thiopurine with allopurinol therapy, but not for AZAm (see next paragraphs).

Two well-known randomized, double-blind trials evaluated the efficacy of $2.5 \mathrm{mg} / \mathrm{kg} /$ day AZAm: steroid-free remission was seen in 30\% (51/170) of Crohn's patients at 6 months [32] and 24\% (18/76) of ulcerative colitis patients at 4 months [33]. The lower response rates compared to our study are likely due to the fact that endpoints 


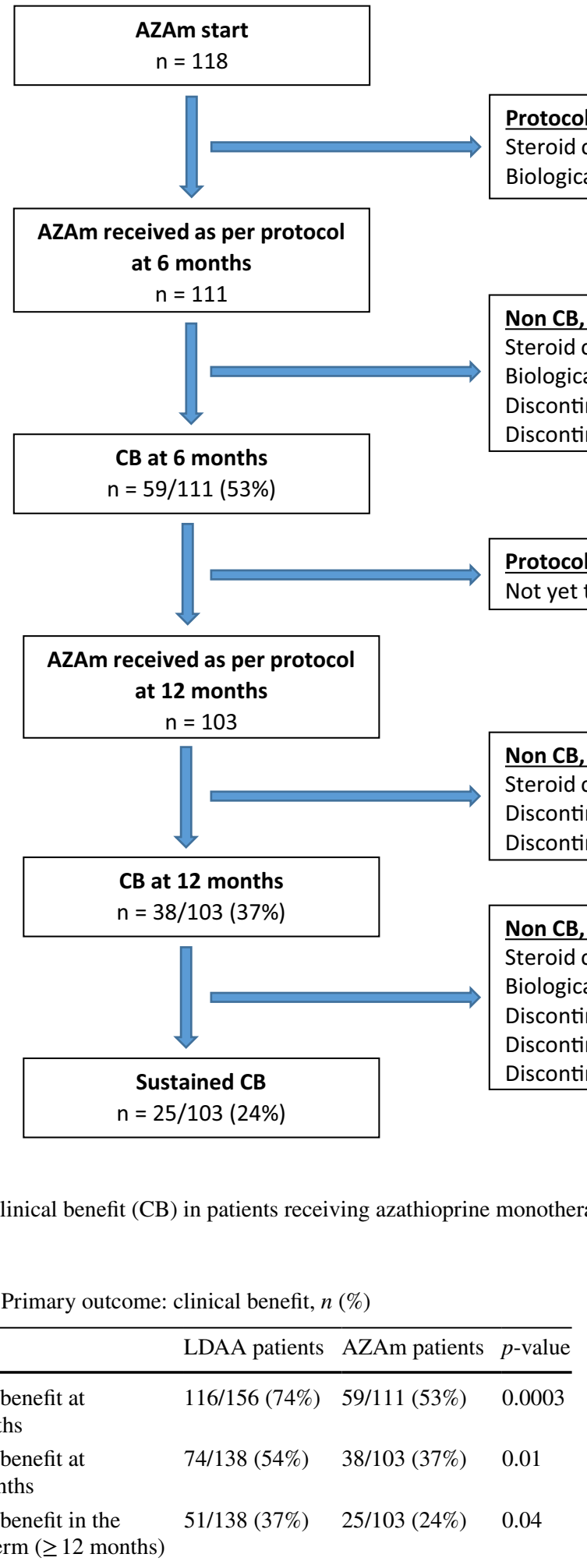

Table 2 Primary outcome: clinical benefit, $n(\%)$

\begin{tabular}{lclc}
\hline & LDAA patients & AZAm patients & $p$-value \\
\hline $\begin{array}{l}\text { Clinical benefit at } \\
6 \text { months }\end{array}$ & $116 / 156(74 \%)$ & $59 / 111(53 \%)$ & 0.0003 \\
$\begin{array}{l}\text { Clinical benefit at } \\
12 \text { months }\end{array}$ & $74 / 138(54 \%)$ & $38 / 103(37 \%)$ & 0.01 \\
$\begin{array}{c}\text { Clinical benefit in the } \\
\text { long-term ( } \geq 12 \text { months) }\end{array}$ & $51 / 138(37 \%)$ & $25 / 103(24 \%)$ & 0.04 \\
\hline
\end{tabular}

of a randomized trial are more difficult to achieve, and that intention-to-treat analysis was used (while we used per-protocol analysis). Recently, long-term effective thiopurine monotherapy was observed in 4995/11928 (42\%) subjects, but analysis did not include initiation of steroids [6].

The high incidence of intolerance to AZAm in our study is in keeping with other studies, in which $30-40 \%$ of patients withdrew AZAm due to adverse events [11, 12, 34, 35]. Additionally, it is worth noting in these studies that tolerability came at the expense of a sub-therapeutic AZA dose. In our study inability to tolerate an effective AZAm dose (i.e. $2.5 \mathrm{mg} / \mathrm{kg} / \mathrm{day}$ for patients with normal TPMT activity [36]) was also observed: only a median dose of $1.83 \mathrm{mg} /$ $\mathrm{kg} / \mathrm{day}$ was achieved, which is $73 \%$ of the effective dose. Conversely, LDAA could be initiated and maintained at the target dose in most patients, with a better response rate. Hence, using LDAA as first-line therapy in clinical practice (instead of AZAm) would likely reduce periods of active 
Fig. 4 Kaplan Meier survival curve of clinical benefit (CB) in patients treated with azathioprine monotherapy (AZAm) and low-dose azathioprine with allopurinol (LDAA). A statistical difference between the curves was found $(p=0.003)$
Table 3 Secondary outcomes, $n(\%)$

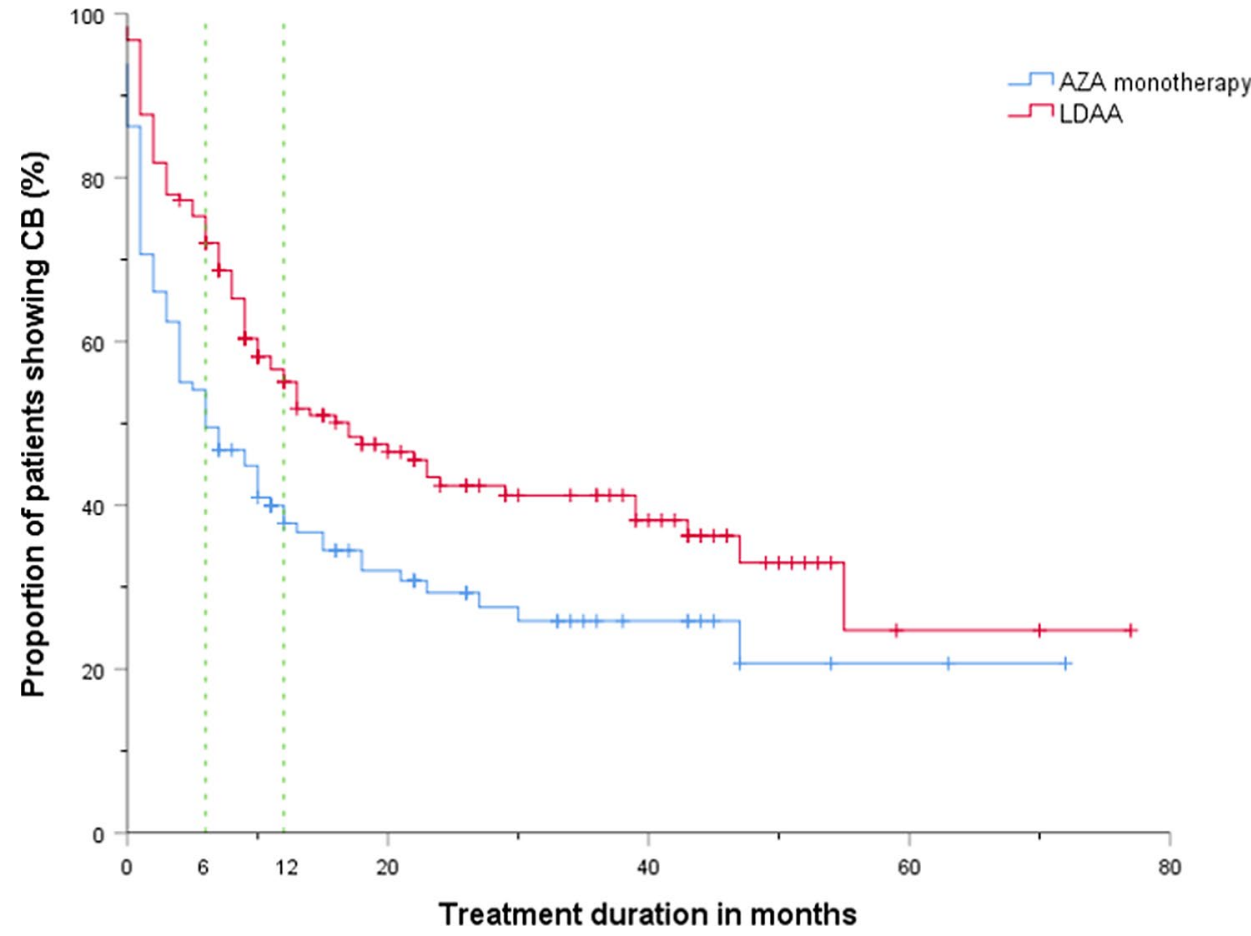

\begin{tabular}{lllllll}
\multicolumn{1}{l}{ at risk } & & & & & & \\
LDAA & 154 & 115 & 73 & 50 & 23 & 2 \\
AZAm & 109 & 59 & 37 & 26 & 9 & 2
\end{tabular}

\begin{tabular}{llll}
\hline & LDAA patients & AZAm patients & $p$-value \\
\hline Steroid withdrawal within 6 months & $44 / 47(94 \%)$ & $27 / 31(87 \%)$ & 0.33 \\
Calcineurin inhibitor withdrawal & $4 / 6(67 \%)$ & $0 / 2(0 \%)$ & 0.10 \\
Anti-tumour necrosis factor withdrawal $^{\mathrm{a}}$ & $4 / 13(31 \%)$ & $0 / 3(0 \%)$ & 0.27 \\
$\mathrm{SCCAI} \leq 2$ at last review & $37 / 53(70 \%)$ & $24 / 38(63 \%)$ & 0.51 \\
$\mathrm{HBI} \leq 4$ at last review & $32 / 53(60 \%)$ & $21 / 40(53 \%)$ & 0.45 \\
$\mathrm{CRP}$ entire treatment duration: & & & \\
$\leq 10 \mathrm{mg} / \mathrm{L}$ & $93 / 158(59 \%)$ & $59 / 94(63 \%)$ & 0.54 \\
$\leq 5 \mathrm{mg} / \mathrm{L}$ & $65 / 158(41 \%)$ & $40 / 94(43 \%)$ & 0.83 \\
\hline
\end{tabular}

$L D A A$ low-dose azathioprine with allopurinol, AZAm azathioprine monotherapy, SCCAI Simple Clinical Colitis Activity Index, $H B I$ Harvey-Bradshaw Index, $C R P$ C-reactive protein

Disease activity scores (SCCAI for ulcerative colitis or HBI for Crohn's disease) were documented in the patients' medical records during follow-up in 106/166 (64\%) LDAA and 78/118 (66\%) AZAm patients; CRP values were available in 158 (95\%) and 94 (80\%) patients, respectively

Eight LDAA patients and 13 AZAm patients could not be assessed for rate of steroid, calcineurin inhibitor or anti-tumour necrosis factor withdrawal, due to insufficient documentation or cessation of thiopurine therapy within 6 months (only applicable for steroid withdrawal)

${ }^{a}$ Biologic therapy could be tapered off in 2/3 LDAA patients (but not in the single AZAm patient) using biologics prior to thiopurine initiation. Of the patients who commenced thiopurine and biologic therapy simultaneously because of a top-down therapeutic approach, 2/10 LDAA and 0/2 AZAm patients could discontinue biologic during follow-up

disease, improve patient experience and eliminate the time and costs associated with managing poor treatment effectiveness and intolerance.
In a previous study, positive response to low-dose thiopurine with allopurinol (AZA in $>50 \%$ of patients, 
Table 4 Adverse events, $n$ (\% of entire cohort)

\begin{tabular}{|c|c|c|c|}
\hline & $\begin{array}{l}\text { LDAA patients } \\
(n=166)\end{array}$ & $\begin{array}{l}\text { AZAm patients } \\
(n=118)\end{array}$ & $p$-value \\
\hline $\begin{array}{l}\text { Patients discontinuing treatment due to adverse } \\
\text { events }\end{array}$ & $43(26 \%)$ & $53(45 \%)$ & 0.001 \\
\hline Hepatotoxicity & $2(1 \%)$ & $3(3 \%)$ & 0.40 \\
\hline Elevated LFTs without hepatotoxicity & $1(0.6 \%)$ & $2(2 \%)$ & 0.37 \\
\hline Myelotoxicity & $3(2 \%)$ & $5(4 \%)$ & 0.22 \\
\hline Leukopenia & $2(1 \%)$ & $3(3 \%)$ & - \\
\hline Thrombocytopenia & $1(0.6 \%)$ & $1(0.8 \%)$ & - \\
\hline Leukopenia and thrombocytopenia & 0 & $1(0.8 \%)$ & - \\
\hline Gastro-intestinal complaints & $22(13 \%)$ & $22(19 \%)$ & 0.22 \\
\hline Fatigue & $8(5 \%)$ & $6(5 \%)$ & 0.92 \\
\hline Dizziness & $6(4 \%)$ & $5(4 \%)$ & 0.79 \\
\hline Arthralgia & $5(3 \%)$ & $3(3 \%)$ & 0.81 \\
\hline Alopecia & $3(2 \%)$ & 0 & 0.14 \\
\hline Pancreatitis & $3(2 \%)$ & $4(3 \%)$ & 0.40 \\
\hline Malaise & $2(1 \%)$ & $4(3 \%)$ & 0.21 \\
\hline Headache & $2(1 \%)$ & $6(5 \%)$ & 0.05 \\
\hline Rash & 0 & $4(3 \%)$ & 0.02 \\
\hline Serious infection & $1(0.6 \%)$ & $1(0.8 \%)$ & 0.81 \\
\hline Malignancy & 0 & 0 & - \\
\hline Unknown & $2(1 \%)$ & $2(2 \%)$ & - \\
\hline Other & $5(3 \%)$ & $11(9 \%)$ & - \\
\hline Patients experiencing adverse events & $80(48 \%)$ & $68(58 \%)$ & 0.12 \\
\hline Hepatotoxicity & $11(7 \%)$ & $8(7 \%)$ & 0.96 \\
\hline Elevated LFTs without hepatotoxicity & $13(8 \%)$ & $10(8 \%)$ & 0.84 \\
\hline Myelotoxicity & $18(11 \%)$ & $11(9 \%)$ & 0.17 \\
\hline Leukopenia & $9(5 \%)$ & $7(6 \%)$ & - \\
\hline Thrombocytopenia & $6(4 \%)$ & $1(0.9 \%)$ & - \\
\hline Leukopenia and thrombocytopenia & $3(2 \%)$ & $3(3 \%)$ & - \\
\hline Gastro-intestinal complaints & $24(15 \%)$ & $24(20 \%)$ & 0.19 \\
\hline Fatigue & $12(7 \%)$ & $7(6 \%)$ & 0.67 \\
\hline Dizziness & $6(4 \%)$ & $6(5 \%)$ & 0.54 \\
\hline Arthralgia & $5(3 \%)$ & $4(3 \%)$ & 0.86 \\
\hline Alopecia & $3(2 \%)$ & 0 & 0.14 \\
\hline Pancreatitis & $3(2 \%)$ & $4(3 \%)$ & 0.40 \\
\hline Malaise & $3(2 \%)$ & $5(4 \%)$ & 0.22 \\
\hline Headache & $5(3 \%)$ & $6(5 \%)$ & 0.37 \\
\hline Rash & 0 & $4(3 \%)$ & 0.02 \\
\hline Serious infection & $1(0.6 \%)^{\mathrm{a}}$ & $1(0.9 \%)^{\mathrm{b}}$ & 0.81 \\
\hline Malignancy & 0 & 0 & - \\
\hline Unknown & $3(2 \%)$ & $2(2 \%)$ & - \\
\hline Other & $7(4 \%)$ & $12(10 \%)$ & - \\
\hline
\end{tabular}

LDAA low-dose azathioprine with allopurinol, AZAm azathioprine monotherapy, LFTs liver function tests ${ }^{\text {a }}$ Respiratory tract infection

${ }^{\mathrm{b}} \mathrm{CMV}$ reactivation mercaptopurine in the rest) at 12 months was seen in $62 \%$ of patients failing thiopurine monotherapy and/or showing hypermethylation [25]. Another study showed thiopurineallopurinol continuation in 78\% $(n=145)$ and $66 \%(n=83)$ of patients at 12 months and 2 years, respectively [37]. The possible explanation for the greater effectiveness in their study might be a twofold lower incidence of patients with active disease at initiation, and that initiation of steroids, 
biologics or surgery was not considered treatment failure. In a recent randomized trial, $73 \%$ of patients on thiopurine and allopurinol (AZA in 50\%) were in steroid-free remission at 6 months according to per-protocol analysis [20]. Lastly, another study showed clinical benefit during at least 12 months in $38 \%$ of the optimized thiopurine cohort (co-administration of allopurinol in 40\%) [23]. The results of these studies are in line with our outcomes.

To date, effectiveness of thiopurine-allopurinol co-therapy in thiopurine-naive patients has only been assessed by our study group [24]. An improvement in IBD activity scores in the absence of steroid use was observed in $79 / 110(72 \%)$ patients after a median treatment duration of 12 months [24].

Our study has limitations which need to be acknowledged. First, allocation to LDAA or AZAm was not randomized. However, collected baseline characteristics in both cohorts were very similar. Another limitation is the retrospective design, which could have led to information bias. The influence of this was minimized by comparing two treatment groups, with a single researcher collecting all of the data. A further limitation concerns sampling bias, as the cohorts were restricted to patients in a single centre. Finally, we were not able to comment on mucosal response or draw firm conclusions regarding CRP level and IBD activity scores due to incomplete data.

Although myelotoxicity is a common concern during LDAA therapy, only 12 (7\%) LDAA patients developed leukopenia during a median follow-up of 25 months. This incidence is comparable to our AZAm cohort, to other thiopurine monotherapy studies $[12,38]$, and also to two large studies using low doses of AZA during co-therapy [26, 37]. The lack of correlation between myelotoxicity and heterozygous TPMT activity in our study can be explained by dose adjustment for weight and TPMT activity.

Elevated LFTs occurred in 14\% (24/166) of LDAA and $15 \%$ (18/118) of AZAm patients during our study, of which $7 \%$ of both cohorts had hepatotoxicity. Nine of twenty-four patients with elevated LFTs in the LDAA cohort had a preexisting liver disorder, which is the likely explanation in these patients. In contrast, the liver injury in the AZAm patients was more likely to be drug-induced as only one patient had a pre-existing liver condition. Previous studies described hepatotoxicity in 5\% of LDAA patients [24] and de-novo elevated LFTs in 14\% [37], which is in line with our results. Interestingly, we showed that increasing the allopurinol dose can be a beneficial strategy to treat hepatotoxicity. On increasing allopurinol to $200-300 \mathrm{mg} /$ day, LFTs fell significantly in $5 / 6$ patients and stabilized in a patient with primary sclerosing cholangitis. These findings are in concordance with our previous study, in which an escalated allopurinol dose of $300 \mathrm{mg}$ /day led to normalization of LFTs in $8 / 11$ patients, with the remaining three patients having primary sclerosing cholangitis [24]. The biochemical mechanism for this phenomenon may be that allopurinol reduces methylated metabolites and reactive oxidative free radicals, both of which are thought to be hepatotoxic [19, 29, 39].

Although myelotoxicity and elevated LFTs were relatively common in our study, they only resulted in LDAA withdrawal in $2 \%$ for either abnormality. Recently, Kreijne et al. reported the same phenomenon in a cohort of 221 patients: myelotoxicity and elevated LFTs led to LDAA cessation in only $4 \%$ and $1 \%$, respectively [37].

In conclusion, this large study demonstrates that optimization of AZA is needed, as poor outcomes were observed in our AZAm cohort. We have shown that first-line coadministration of allopurinol is a long-term effective and safe optimization strategy, even without metabolite monitoring. Although patients may still experience mild laboratory abnormality, we confirmed that myelotoxicity and elevated liver enzymes rarely necessitate LDAA withdrawal if closely monitored and adequately treated. Using LDAA as first-line therapy for IBD (rather than AZAm) would likely reduce periods of active disease, improve patient experience and reduce the number of patients escalated to more expensive treatments like biologics. Hence, LDAA may be considered as standard first-line immunosuppressive.

Supplementary Information The online version contains supplementary material available at https://doi.org/10.1007/s10620-021-07273-y.

Funding This study was not funded.

\section{Declarations}

Conflict of interest ELSA van Liere and AB Bayoumy received unrestricted travel grants from Thimotheus Consult. CJJ Mulder has served as consultant for HLW Pharma BV, Douglas Pharma, Arega and TEVA Pharma BV. NKH de Boer has served as consultant and principal investigator for TEVA Pharma BV and Takeda. He has served as a speaker for AbbVie and MSD and has received unrestricted research grants from Dr. Falk, TEVA Pharma BV and Takeda. AR Ansari has served as consultant and speaker for Dr. Falk. He has received unrestricted research grants from Janssen Cilag and MSD. SHC Anderson, B Warner, B Hayee, BA Mateen and JD Nolan declare that they have no conflict of interest.

Open Access This article is licensed under a Creative Commons Attribution-NonCommercial 4.0 International License, which permits any non-commercial use, sharing, adaptation, distribution and reproduction in any medium or format, as long as you give appropriate credit to the original author(s) and the source, provide a link to the Creative Commons licence, and indicate if changes were made. The images or other third party material in this article are included in the article's Creative Commons licence, unless indicated otherwise in a credit line to the material. If material is not included in the article's Creative Commons licence and your intended use is not permitted by statutory regulation or exceeds the permitted use, you will need to obtain permission directly from the copyright holder. To view a copy of this licence, visit http://creativecommons.org/licenses/by-nc/4.0/. 


\section{References}

1. Harbord $\mathrm{M}$ et al. Third European evidence-based consensus on diagnosis and management of ulcerative colitis: Part 2: current management. J Crohn's Colitis. 2017;11:769-784.

2. Torres $\mathbf{J}$ et al. ECCO guidelines on therapeutics in Crohn's disease: Medical treatment. J Crohns Colitis. 2020;14:4-22.

3. Camus M et al. Long-term outcome of patients with Crohn's disease who respond to azathioprine. Clin Gastroenterol Hepatol. 2013;11:389-394.

4. Chatu $\mathrm{S}$ et al. The role of thiopurines in reducing the need for surgical resection in Crohn's disease: a systematic review and meta-analysis. Am J Gastroenterol. 2014;109:23-34 (quiz 35).

5. Ramadas AV et al. Natural history of Crohn's disease in a population-based cohort from Cardiff (1986-2003): a study of changes in medical treatment and surgical resection rates. Gut. 2010;59:1200-1206.

6. Stournaras E et al. Thiopurine monotherapy is effective in ulcerative colitis but significantly less so in Crohn's disease: long-term outcomes for 11928 patients in the UK inflammatory bowel disease bioresource. Gut. 2021;70:677-686.

7. Zhu $\mathrm{Z}$ et al. Reduced risk of inflammatory bowel disease-associated colorectal neoplasia with use of thiopurines: a systematic review and meta-analysis. J Crohns Colitis. 2018;12:546-558.

8. Christensen LA et al. Azathioprine treatment during lactation. Aliment Pharmacol Ther. 2008;28:1209-1213.

9. Coelho $\mathrm{J}$ et al. Pregnancy outcome in patients with inflammatory bowel disease treated with thiopurines: cohort from the CESAME Study. Gut. 2011;60:198-203.

10. Lamb CA et al. British Society of Gastroenterology consensus guidelines on the management of inflammatory bowel disease in adults. Gut. 2019;68:s1-s106.

11. Ansari A et al. Prospective evaluation of the pharmacogenetics of azathioprine in the treatment of inflammatory bowel disease. Aliment Pharmacol Ther. 2008;28:973-983.

12. Sood $\mathrm{R}$ et al. Long-term efficacy and safety of azathioprine in ulcerative colitis. J Crohns Colitis. 2015;9:191-197.

13. Jharap B et al. Thiopurine therapy in inflammatory bowel disease patients: analyses of two 8-year intercept cohorts. Inflamm Bowel Dis. 2010;16:1541-1549.

14. Mazor Y et al. Adalimumab drug and antibody levels as predictors of clinical and laboratory response in patients with Crohn's disease. Aliment Pharmacol Ther. 2014;40:620-628.

15. Buisson $A$ et al. The extra burden of infliximab infusions in inflammatory bowel disease. Inflamm Bowel Dis. 2013;19:2464-2467.

16. Lichtenstein $\mathrm{L}$ et al. Infliximab-related infusion reactions: systematic review. J Crohns Colitis. 2015;9:806-815.

17. van der Valk ME et al. Healthcare costs of inflammatory bowel disease have shifted from hospitalisation and surgery towards anti-TNFalpha therapy: results from the COIN study. Gut. 2014;63:72-79.

18. Bayoumy AB, de Boer NKH, Mulder CJJ. Management of Crohn disease. JAMA. 2021;325:1793-1794.

19. Sparrow MP et al. Allopurinol safely and effectively optimizes tioguanine metabolites in inflammatory bowel disease patients not responding to azathioprine and mercaptopurine. Aliment Pharmacol Ther. 2005;22:441-446.

20. Friedman $A B$ et al. Randomised clinical trial: efficacy, safety and dosage of adjunctive allopurinol in azathioprine/mercaptopurine nonresponders (AAA Study). Aliment Pharmacol Ther. 2018;47:1092-1102.

21. Hoentjen $\mathrm{F}$ et al. Safety and effectiveness of long-term allopurinol-thiopurine maintenance treatment in inflammatory bowel disease. Inflamm Bowel Dis. 2013;19:363-369.
22. Leung $\mathrm{Y}$ et al. Long term efficacy and safety of allopurinol and azathioprine or 6-mercaptopurine in patients with inflammatory bowel disease. J Crohns Colitis. 2009;3:162-167.

23. Meijer B et al. Optimizing thiopurine therapy in inflammatory bowel disease among 2 real-life intercept cohorts: Effect of allopurinol comedication? Inflamm Bowel Dis. 2017;23:2011-2017.

24. Pavlidis $\mathbf{P}$ et al. Long-term safety and efficacy of low-dose azathioprine and allopurinol cotherapy in inflammatory bowel disease: a large observational study. Inflamm Bowel Dis. 2016;22:1639-1646.

25. Smith MA et al. Optimising outcome on thiopurines in inflammatory bowel disease by co-prescription of allopurinol. J Crohns Colitis. 2012;6:905-912.

26. Vasudevan A et al. Low-dose thiopurine with allopurinol cotherapy overcomes thiopurine intolerance and allows thiopurine continuation in inflammatory bowel disease. Dig Liver Dis. 2018;50:682-688.

27. Cushing K, Higgins PDR. Management of Crohn disease: a review. JAMA. 2021;325:69-80.

28. Haines ML et al. Clinical usefulness of therapeutic drug monitoring of thiopurines in patients with inadequately controlled inflammatory bowel disease. Inflamm Bowel Dis. 2011;17:1301-1307.

29. Shaye OA et al. Hepatotoxicity of 6-mercaptopurine (6-MP) and Azathioprine (AZA) in adult IBD patients. Am J Gastroenterol. 2007;102:2488-2494.

30. Reinshagen $\mathrm{M}$ et al. 6-Thioguanine nucleotide-adapted azathioprine therapy does not lead to higher remission rates than standard therapy in chronic active crohn disease: results from a randomized, controlled, open trial. Clin Chem. 2007;53:1306-1314.

31. Chande $\mathrm{N}$ et al. Azathioprine or 6-mercaptopurine for induction of remission in Crohn's disease. Cochrane Database Syst Rev. 2016;10:CD000545.

32. Colombel JF et al. Infliximab, azathioprine, or combination therapy for Crohn's disease. N Engl J Med. 2010;362:1383-1395.

33. Panaccione $\mathrm{R}$ et al. Combination therapy with infliximab and azathioprine is superior to monotherapy with either agent in ulcerative colitis. Gastroenterology. 2014;146:392-400 e3.

34. Hindorf $U$ et al. Adverse events leading to modification of therapy in a large cohort of patients with inflammatory bowel disease. Aliment Pharmacol Ther. 2006;24:331-342.

35. Verstockt B et al. Thiopurine monotherapy has a limited place in treatment of patients with mild-to-moderate Crohn's disease. Gut. 2020;70:1416-1418.

36. Prefontaine E et al. Azathioprine or 6-mercaptopurine for maintenance of remission in Crohn's disease. Cochrane Database Syst Rev. 2009;1:CD000067.

37. Kreijne JE et al. Real-life study of safety of thiopurine-allopurinol combination therapy in inflammatory bowel disease: myelotoxicity and hepatotoxicity rarely affect maintenance treatment. Aliment Pharmacol Ther. 2019;50:407-415.

38. Teml A et al. Thiopurine treatment in inflammatory bowel disease: clinical pharmacology and implication of pharmacogenetically guided dosing. Clin Pharmacokinet. 2007;46:187-208.

39. Duley JA, Florin TH. Thiopurine therapies: problems, complexities, and progress with monitoring thioguanine nucleotides. Ther Drug Monit. 2005;27:647-654.

Publisher's Note Springer Nature remains neutral with regard to jurisdictional claims in published maps and institutional affiliations. 


\section{Authors and Affiliations}

Elsa L. S. A. van Liere ${ }^{1,2}$ (D) Ahmed B. Bayoumy ${ }^{1} \cdot$ Chris J. J. Mulder ${ }^{3} \cdot$ Ben Warner $^{4} \cdot$ Bu Hayee $^{5} \cdot$ Bilal A. Mateen $^{5}$. Jonathan D. Nolan ${ }^{2}$ Nanne K. H. de Boer ${ }^{3}$. Simon H. C. Anderson ${ }^{4}$ Azhar R. Ansari²

Ahmed B. Bayoumy

a.b.bayoumy@amsterdamumc.nl

Chris J. J. Mulder

cjmulder@amsterdamumc.nl

Ben Warner

b.warner@nhs.net

Bu Hayee

b.hayee@nhs.net

Bilal A. Mateen

bilal.mateen@nhs.net

Jonathan D. Nolan

jonathan.nolan2@nhs.net

Nanne K. H. de Boer

khn.deboer@amsterdamumc.nl

Simon H. C. Anderson

Simon.Anderson@gstt.nhs.uk
Azhar R. Ansari

azharansari@nhs.net

1 Faculty of Medicine, Amsterdam UMC, VU University Medical Centre, De Boelelaan 1118, 1081 HZ Amsterdam, The Netherlands

2 Department of Gastroenterology and Hepatology, Surrey and Sussex NHS, Easy Surrey Hospital, Redhill RH1 5RH, UK

3 Department of Gastroenterology and Hepatology, AGEM Research Institute, Amsterdam UMC, VU University Medical Centre, 1081 HZ Amsterdam, The Netherlands

4 Department of Gastroenterology and Hepatology, Guy's and St Thomas' NHS Foundation Trust, London SE1 7EH, UK

5 IBD Service, King's College Hospital NHS Foundation Trust, London SE5 9RS, UK 